\title{
Applying Reach in Direct Manipulation User Interfaces
}

\author{
Aaron Toney and Bruce H. Thomas \\ School of Computer \& Information Science, \\ University of South Australia \\ aaron.toney@hhhh.org,bruce.thomas@unisa.edu.au
}

\begin{abstract}
The HCI community currently faces the problem of making tangible user interfaces actively responsive to their user's current physical context. This paper explores the context of direct manipulation user interfaces for large horizontal interactive displays. Knowledge of users' reach provides direct manipulation user interfaces with a powerful tool for contextualizing and predicting user action. This paper introduces users' reach as a formal way to predict the previously observed phenomena of workspace segmentation and territoriality. By creating models of "reach-ability", reach probability surfaces can be generated which further explain the impact on workspace usage of the shape, height, and position of the workspace. As the presented techniques build on formal qualitative and mathematical models of reach, they lend themselves particularly well to an algorithmic implementation suited to driving complex user interface behaviour. This paper presents the results of an initial user study to determine the accuracy of these predictions and their underlying hypotheses about reaches role in shaping workspace usage.
\end{abstract}

\section{Author Keywords}

Reach, Reach envelope, OTRE, Reach probability surface, Tangible, User interface

\section{ACM Classification Keywords}

H5.2 [Information interfaces and presentation]: User Interfaces. - Graphical user interfaces.

\section{INTRODUCTION}

Applying anthropometric observations made about the user enables the creation of user interfaces that are responsive to their user's physical context. This paper explores the role of reach within the context of direct manipulation user interfaces for large horizontal interactive displays. In this context reach refers to the maximum extension of the arm at which the user can perform a manipulation. This paper introduces applying models of reach, derivable from easily observable anthropometric characteristics [1], to roughly predict working surface usage patterns.

For the individual, depth of reach is shown to predict segmentation of the working plane into working and storage areas. For multiple collaborating users, regions of overlapping reach are shown to predict the formation,

OZCHI 2006, November 20-24, 2006, Sydney, Australia. Copyright the author(s) and CHISIG

Additional copies are available at the ACM Digital Library (http://portal.acm.org/dl.cfm) or ordered from the CHISIG secretary (secretary@chisig.org)

OZCHI 2006 Proceedings ISBN: 1-59593-545-2 size, and shape of shared group spaces. In both contexts applied models of reach predicts the rough impact of such variables as working surface size, height, shape, and number of users on the working surface's utilization. The authors performed a 16 person user study to empirically test the predictions generated by applying reach models.

\section{THE REACH ENVELOPE}

The On Table Reach Envelope (OTRE) represents the boundary of the set of all reachable points $\left(S_{R}\right)$. This section will present the OTRE from an individual and the use of OTRE for the segmentation of a work surfaces for multiple collocated users.

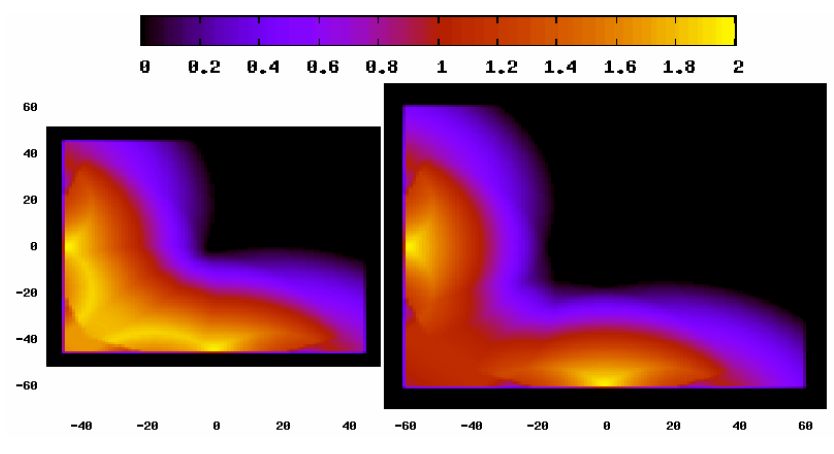

Figure 1 Predicted on-table reach probability for adjacently seated participant at $90 \mathrm{~cm}$, and $120 \mathrm{~cm}$ square tables

\section{Modeling Reach}

The authors have previously noted that qualitative models of reach used in industrial design and ergonomics provide descriptions of an individuals reach that are suitable for use in user interface design $[1,2]$. The simplest mathematical model for the reach envelope is a hemispherical model, the Zone of Convenient Reach (ZCR). Reachable space for the ZCR is considered to be a hemisphere $\left.\left(\mathrm{R}<\mid P_{\left\{x, y_{\}}-\right.} S_{\{x, y\}}\right) \mid\right)$ centered on the shoulder $\left(S_{\{x, y\}}\right)$ with a radius $(R)$ equal to the extended arm length. The model assumes the arm is of fixed length rotating about the center point of the shoulder.

We have extended the ZCR to provide a simple estimate of "reachability" for space within the reach hemisphere. The extended model was used to generate the simulations in Figure 1, Figure 4, and Figure 5. These figures report the simulated impact of changes in table size, shape, and seating location.

The simulations all assume that there is an ideal working distance $\left(R_{I D E A L}\right)$, and that "reachability" linearly increases with distance from the shoulder rotation point to Reach $_{I D E A L}$, after which point "reachability" decreases to zero at the maximum reach $(R)$. Reachable space is constrained within a maximum and minimum horizontal adduction and abduction angles [3, 4]. These angles are 
used to constrain the model, limiting the motion of the arm to a fraction of the hemispherical shell.

\section{Table Segmentation for Individuals}

The authors hypothesize that working space $\left(S_{W}\right)$ is primarily contained within the most easily reachable areas. Further, the highest usage will occur in the area of bimanual reach $\left(S_{R B}\right)$, while lowest usage will occur in areas reachable only by one hand $\left(S_{R}-S_{R B}\right)$.

Under these hypotheses the region of bimanual reach $S_{R B}$, describes the user's working space, $S_{W}$. These hypotheses predict the previously observed table segmentation into storage and working spaces $[5,6]$ and is supported by the results of our user study. For a given posture, the reachable space $\left(S_{R}\right)$ is by definition the total space reachable by either hand $\left(A_{L} \cup A_{R}\right)$, while an individual's bimanual reach $\left(S_{R B}\right)$ is by definition $\left(A_{L} \cap A_{R}\right)$. With the working space $\left(S_{W}\right)$ equivalent to $S_{R B}$, the storage space $\left(S_{S}\right)$ is seen to be the relative complement of the total reachable space and the working space $\left(S_{R}-S_{R B}\right)$.

\section{Table Segmentation for Co-Located Users}

The authors hypothesize that group spaces are formed in preference to personal spaces. Under this hypothesis the group space $\left(S_{G}\right)$ can be described as the intersection of all users' reachable spaces $\left(\cap S_{R}\right)$, and the co-located working space $\left(S_{W}\right)$ can then be described as the reachable area that is not a group space $\left(S_{R B}-\cap S_{R}\right)$ or $\left(S_{R B}-S_{G}\right)$. These definitions agree with both the observations in the literature $[5,6]$ and the results of the user study presented in this paper.

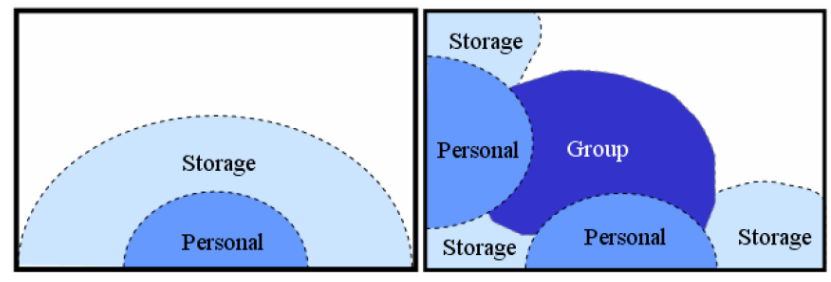

Figure 2 Territories observed by Scott [5]

\section{Motion in Presented OTRE Models}

As users move, the envelopes of both reachable space $\left(S_{R}\right)$ and bimanually reachable space $\left(S_{R B}\right)$, becomes the union of all the user's individual reach envelopes over time; so $S_{R}$ and $S_{R B}$ become $\bigcup_{t} S_{R}$ and $\bigcup_{t} S_{R B}$ respectively. While the total potential group space is increased by user motion, the group space available to all of the users $\left(S_{G A}\right.$ $\left.=\bigcap_{t} S_{G}\right)$ shrink. The total potential group space is increased because of the possible formation of subgroup spaces $\left(S_{G i}\right)$ form within $\left(\bigcup_{t} S_{G}-S_{G A}\right)$.

\section{Comfortable Reach}

Comfortable reach, $S_{R \text {-comfortable, }}$ is the union of the user's physical over their comfortable range of motion, $\mathrm{U}_{\text {comfortable }} S_{R}$. Both in the current and previous work comfortable reach was observed to be highly asymmetric, with comfortable reach significantly greater for the dominant hand [1,7]. Aggregate descriptions of reach in turn reflect this asymmetry. The ZCR model predicts physical not comfortable reach. This is why Figure 1,
Figure 4, and Figure 5, which simulate physical reach, show such a high degree of symmetry. Initial results suggest that an application which can guide the seating locations of its users can arrange the seating to maximize overlapping hand dominance encouraging collaboration.

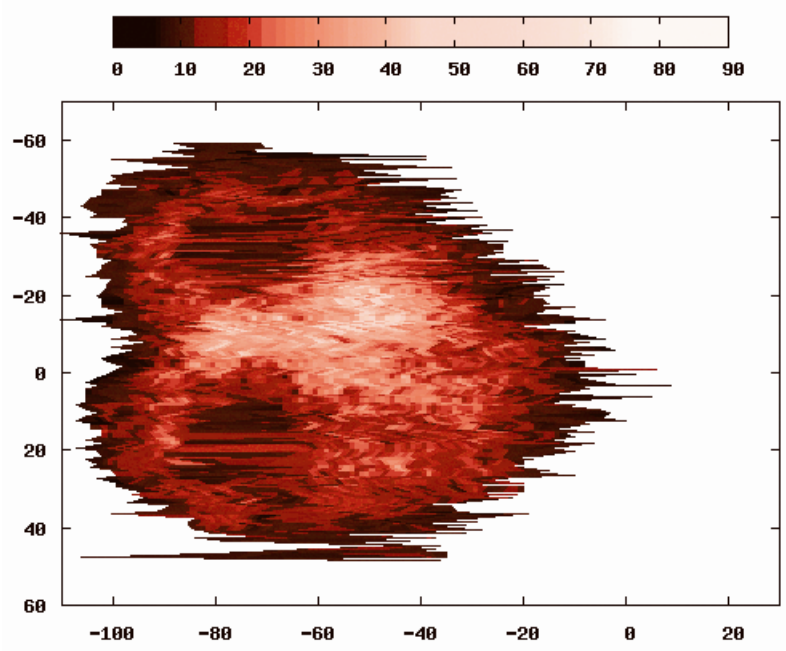

Figure 3 Observed Hand Positions (The user is seated at approximately $(10,-100)$ and facing to the right.)

\section{FORMAL STUDY OF TABLE USAGE}

After a survey of the literature and several informal studies [7], the authors found assembly of LEGOTM models to be a task representative of complex direct manipulation user interfaces with significant acquisition and manipulation components. Sixteen participants were recruited from staff and students in the School of Computer and Information Science at the University of South Australia. Participants were selected with the following three restrictions: no color blindness, no condition limiting their reach, and no conditions preventing comfortable seating for extended periods of time. Two participants were female and two were left handed.

Participants were provided with an assortment of 997 pieces of standard LEGO ${ }^{\mathrm{TM}}$. Mixed in with these pieces were two different LEGO ${ }^{\mathrm{TM}}$ kits, each consisting of less than 150 pieces. Participants were then given the official $\mathrm{LEGO}^{\mathrm{TM}}$ pictographic directions for the $\mathrm{LEGO}^{\mathrm{TM}}$ kits and given 45 minutes in which to assemble the kit. In order to encourage searching and sorting behavior the models chosen were primarily of a single color and all duplicate pieces for either model were removed from the bucket. All of the participants but one reported having previous experience with $\mathrm{LEGO}^{\mathrm{TM}}$. On a scale from 1 to 5, participants self rating of their LEGO ${ }^{\mathrm{TM}}$ ability averaged 3.61 with a standard deviation of 0.59 .

Participants were tested in pairs seated adjacently at a $73 \mathrm{~cm}$ tall, $120 \mathrm{~cm} \times 120 \mathrm{~cm}$ table. The torsos of the participants were centered to the edges of the table. A magnetic sensor from a Polhemus FASTRAK was strapped to the back of the wrists of each participant. These sensors allowed participant hand position to be tracked with sub-centimeter accuracy at a rate of 15 samples per second. 


\section{RESULTS AND DISCUSSION}

The results and discussion are presented in context of different areas of the table the participants employed during the study. The areas presented in this section are as follows: the entire table, edges, corners, storage areas, and where is the "sweet spot". There was a fault in one of the sensors of the Polhemus tracker, and therefore while observations are reported for both users, the quantitative data only reflects observations for a single participant.

\section{The Entire Table}

Table usage was observed to be centered on the sagittal plane of the body. A thresholded spatial histogram of the gathered data is presented in Figure 3. The thresholding removed locations with fewer then five samples. The figure indicates the number of times a participant's hand was recorded as being within each cubic centimeter above the working plane. The spatial histogram shows reach to be an elliptical phenomenon, confirming the hypothesis that highest usage will be in areas of bimanual action $\left(S_{R B}\right)$, and lowest usage in areas reachable only by one hand $\left(S_{R}-S_{R B}\right)$.

\section{Edges}

One of the on table phenomena the authors originally set out to measure was the minimum distance from the edges of the table that participants were comfortable placing artifacts. All participants in the study had at least one of their working or storage piles spread so that it touched an edge of the table.

\section{Corners}

A goal of the study was to examine the impact of corners on the utilization of working surface real-estate in direct manipulation UIs. Corners have previously been observed by Stott et all [5] (Figure 2) as storage spaces on the table. Applying OTREs to predict workspace segmentation disagrees with Scott's observations, predicting that as space reachable by adjacently seated participants corners should form group spaces on the table. The usage observed in the study disagreed with either prediction. During the study participants showed a reluctance to use the corners leaving on average an approximately $20 \mathrm{~cm} \mathrm{x}$ $20 \mathrm{~cm}$ wedge of unused but reachable space at the corner of the table between the two subjects.

\section{Storage and Sorting Areas}

All of the participants eventually fanned out the LEGO over communal group areas on the table so that it could be easily scanned. As participants discovered pieces of possible later use they were moved to storage areas closer to hand. As these areas held a small number of pieces they could be frequently scanned visually and only reached into in order to store or retrieve specific pieces. Areas of low table usage were observed on either side of the working space (Figure 3). These regions were approximately $26 \mathrm{~cm}$ by $12 \mathrm{~cm}$ deep $\left(312 \mathrm{~cm}^{2}\right)$ and corresponded to the storage spaces for specific pieces the participant had previously set aside. The formation of this dead space supports the prediction that the storage space $\left(S_{S}\right)$ will form in areas reachable by only one hand $\left(S_{R}-\right.$ $\left.S_{R B}\right)$.

\section{Where is the "Sweet-Spot?"}

The highest usage area fanned out in front of the users to a mean maximum depth of almost $50 \mathrm{~cm}$. Over $90 \%$ of the recorded table usage was constrained to within an approximately $12 \mathrm{~cm}$ wide and $34 \mathrm{~cm}$ deep $\left(410 \mathrm{~cm}^{2}\right)$ region in front of the participant. This observations show the primary working space size and location to be within the area of bimanual action [1].

The average planar distance between the wrists over the course of the study was $48.97 \mathrm{~cm}$ with a variance of $13.26 \mathrm{~cm}$. This corresponds to an average bounding area for the manipulation area of $1275-3873 \mathrm{~cm}^{2}$ (mean at $2398 \mathrm{~cm}^{2}$ ). During assembly the inward bending of the fingers can act to significantly reduce this distance. These observations support the hypothesis that working space $\left(S_{W}\right)$ is primarily contained within the area of bimanual reach $\left(S_{R B}\right)$.

\section{CONCLUSIONS ABOUT DIRECT MANIPULATION}

We employed simulations of user reach to augment our observed study data with predicted utilization of table workspaces. All the users simulated are anthropometrically representative of the $50^{\text {th }}$ percentile females [4, 8, 9] seated $17 \mathrm{~cm}$ [1] from the tables edge. We simulated users of a single size and gender to separate artifacts of overlapping reach from artifacts arising from a difference in size between simulated users. This extended model was used to generate Figure 1, Figure 4, and Figure 5. The coloring indicates an additive estimate of "reachability" based on the number of overlapping OTREs in a given area.

\section{Reach Shadows}

The authors have previously shown how the torso shadows reachable space [1]. The adduction angle restriction in the extended ZCR model used by the authors represents the torso's shadowing of reach. Objects resting on the working surface also cast shadows in reachable space. Shadows are cast when the user can not reach over or around an object on the table. The users pictured using the prototypes presented in Shen et all [10] and $\mathrm{Wu}$ et al [11] provide examples of how users bring objects, most notably cups and glasses, to the table. As researchers we need to carefully conduct our experiments to minimize confounding caused by artifacts users bring with them to the working surface.

\section{The Impact of Table Size}

Workspace utilization for direct manipulation user interfaces is sensitively dependent on table size[12]. In Figure 4 an increase in the table size by only $10 \mathrm{~cm}$ on a side is shown to cause the loss of the comfortable communal group region $\left(S_{G}=\cap S_{R}\right)$. For the larger table subjects will have to lean forward extending their reach to access the communal areas of the table available to all members of the group.

The simulation in Figure 4 illustrates that increased table size discourages direct physical collaboration between all participants at the table. As the table's size is increased the collaboration space at the center of the table $\left(S_{G}\right.$ or $\cap S_{R}$ ) becomes less accessible. As a result larger tables 
show preference to lateral "shoulder-to-shoulder" collaboration. This effect is seen in the clustering patterns observed by Ryall et all [12] and Scott et all [5].
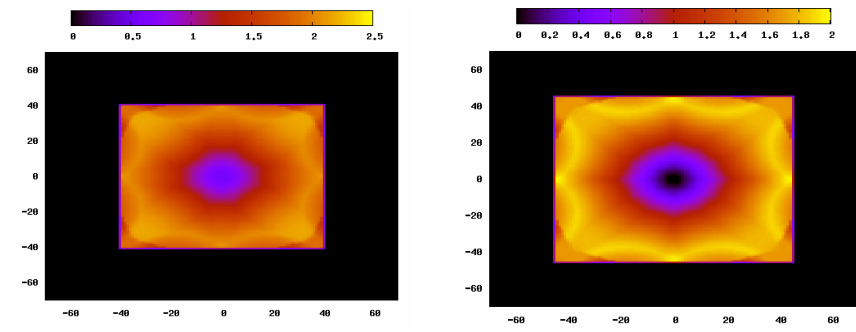

Figure 4 Predicted table usage for four participants seated at $80 \mathrm{~cm}$ and $90 \mathrm{~cm}$ tables

Currently a common research practice is to prototype horizontal interactive displays by mounting large computer monitors and televisions to form the working surface. The resulting workspaces have the same aspect ratio as the display. Figure 1 of both Shen et all [10] and Wu et al [11] show examples of such prototype displays. The aspect ratio means that users will be presented with unequal and very different amounts of on-table realestate. Extreme care should be taken to account for this unequal representation when conducting research using this type of horizontal interactive display.
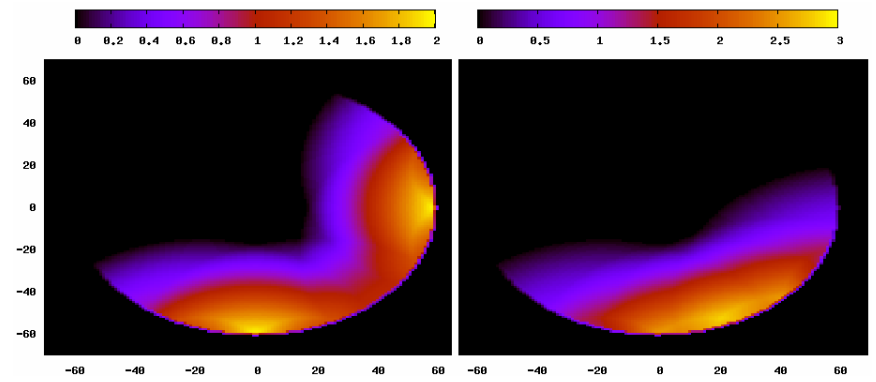

Figure 5 Predicted on-table reach probability for seated participants with an inner sagittal plane angle of $90^{\circ}$

(left), and $45^{\circ}$ (right) square tables

\section{The Impact of Table Shape}

We employed a simulation of user reach to predict usable space in a table workspace. This extended model was used to generate Figure 1, Figure 4, and Figure 5. The coloring indicates an additive estimate of "reachability" based on the number of overlapping OTREs in a given area. A small change in table shape can have a dramatic impact on the usage of the table. For example a curved table edges allow for inner sagittal plane angles that are not even multiples of $90^{\circ}$. As the angle between the sagittal planes of collaborators decreases the total amount of reachable space on the table $\left(U S_{R}\right)$ decreases while the overlapping reachable spaces $\left(\cap S_{R}\right)$ increases; see Figure 5. As a result when users are oriented such that the sagittal plane of their body is normal to the working surface's edge at its closest point, there is more collaborative space between the users and is thus better suited for shoulder to shoulder collaboration then the straight edge.

\section{CONCLUSION}

Currently computer scientists in general think and work mostly in terms of small, on-screen interfaces. This paper explored the context of direct manipulation user interfaces for large horizontal interactive displays. Specifically this paper examined how models of the reach envelope can be used to improve the design of direct manipulation user interfaces. Applied anthropometric characteristics, specifically user reach, were shown to contextualize and predict utilization of the working plane. User reach was shown to be able to explain observed table segmentation into working, storage and group areas. For groups regions of overlapping reach were shown to help explain territoriality and the size and shape of collaborative areas. Additionally reach was shown to be able to assess the impact of table shape, size, and the placement of users on the utilization of a working plane. Application of reach models is a powerful tool enabling the creation of direct manipulation user interfaces that are actively tailored to their users.

\section{REFERENCES}

1. Toney, A. and B.H. Thomas. Modeling Reach for use in User Interface Design. in AUIC. 2007. Victoria, Australia.

2. Toney, A. and B.H. Thomas. Considering Reach in Tangible and Table Top Design. in First IEEE International Workshop on Horizontal Interactive Human-Computer Systems. 2006. Adelaide, South Australia.

3. Wang, Y., B. Das, and A.K. Sengupta, Normal horizontal working area: the concept of inner boundary. Ergonomics, 1999. 42(4): p. 638-646.

4. NASA, Man-Systems Integration Standards, Revision B. 1995.

5. Stacey D. Scott, M. Sheelagh T. Carpendale, and K.M. Inkpen. Territoriality in Collaborative Tabletop Workspaces. in CSCW. 2003. Chicago, Illinois, USA.: ACM.

6. Scott, S.D., Territoriality in Collaborative Tabletop Workspaces, in Department of Computer Science. 2005, University of Calgary: Alberta.

7. Toney, A. and B.H. Thomas. Considering Reach in Tangible and Tabltop Design. in TableTop. 2005. Adelaide: IEEE.

8. Donelson, S.M. and C. Gordon, 1995 Matched Anthropometric database of U.S.Marine Corps Personnel: Summary Statistics. 1996, United States Army Soldier Systems Command.

9. Paquette, S.P., et al., A Supplement to the 1995 matched anthropometric database of U.S. Marine corps personnel: summary statistics. 1997, United States Army: Natick, Massachusetts.

10. Shen, C., et al. DiamondSpin: An Extensible Toolkit for Around-the-Table Interaction. in CHI. 2004. Vienna, Austria: ACM.

11. $\mathrm{Wu}, \mathrm{M}$., et al. Gesture Registration, Relaxation, and Reuse for Multi-Point Direct-Touch Surfaces. in TableTop. 2006. Adelaide, Australia.

12. Ryall, K., et al. Exploring the Effects of Group Size and Table Size on Interactions with Tabletop SharedDisplay Groupware. in CSCW. 2004. Chicago, Illinois, USA. 